\title{
Evolution of Space Shuttle Range Safety Ascent Flight Envelope Design
}

\author{
Joan Brewer ${ }^{1}$ \\ United Space Alliance, LLC, Houston, TX 77058 \\ Jerel Davis ${ }^{2}$ \\ United Space Alliance, LLC, Houston, TX 77058 \\ Christopher Glenn ${ }^{3}$ \\ NASA Johnson Space Center, Houston, TX 77058
}

\begin{abstract}
For every space vehicle launch from the Eastern Range in Florida, the range user must provide specific Range Safety (RS) data products to the Air Force's $45^{\text {th }}$ Space Wing in order to obtain flight plan approval. One of these data products is a set of RS ascent flight envelope trajectories that define the normal operating region of the vehicle during powered flight. With the Shuttle Program launching 135 manned missions over a 30-year period, 135 envelope sets were delivered to the range. During this time, the envelope methodology and design process evolved to support mission changes, maintain high data quality, and reduce costs. The purpose of this document is to outline the shuttle envelope design evolution and capture the lessons learned that could apply to future spaceflight endeavors.
\end{abstract}

\section{Introduction}

$\mathrm{T}_{\mathrm{p}}^{\mathrm{H}}$ HE purpose of Range Safety (RS) is to minimize the risk to public life, health, and property associated with the potential hazards of launching a vehicle into space. NASA's Space Shuttle Program (SSP) launched its vehicles from Kennedy Space Center's Launch Complex 39 on the Eastern Range (ER) in Florida. The Air Force $45^{\text {th }}$ Space Wing (45SW) operates these ER launch pads and worked with NASA to support RS measures for each Shuttle launch.

One of the ways the 45SW carries out its RS function is to monitor a vehicle's real-time ascent trajectory on visual displays located at Florida's Cape Canaveral Air Force Station. These RS displays allow the 45SW to see if the vehicle is traveling as planned in horizontal and vertical plane views. They also provide information required to protect critical geographic areas and population centers that are defined prior to launch. In order to build the displays for a given launch, vehicle ascent trajectory data are required of the range user in accordance with specific requirements documented in Air Force Space Command Manual 91-710 (AFSPCMAN 91-710)*. Ascent trajectory data are only one part of the complete RS trajectory data package required for each launch.

For the Shuttle Program, the timing of the ascent data generation process was important as there was a specific time window in which to build the data. The window began at the end of the Shuttle ascent flight design process when the most current mission input data were available. The window ended at the launch-minus date specified by the $45 \mathrm{SW}$ for the given mission or program. It was critical that the data be correct and delivered on schedule in order for the range to have time to build their displays and to grant NASA flight plan approval for the launch.

The ascent data requirements include the vehicle's nominal ascent trajectory and a set of 3-sigma ascent trajectory flight envelopes. The envelopes define the normal flying region of the vehicle's position from lift-off to the end of powered flight. For a normally-operating vehicle during ascent, 99.865 percent of all trajectories would be expected to fly within these 3-sigma envelopes.

\footnotetext{
${ }^{1}$ Aerospace Engineer, Ascent/Descent Flight Design, 600 Gemini Ave, USH-482L

${ }^{2}$ Mechanical Engineer, Ascent/Descent Flight Design, 600 Gemini Ave, USH-482L

${ }^{3}$ Aerospace Engineer, Flight Dynamics Division - Ascent Analysis, 2101 NASA Parkway, DM44
} 
Nominal envelopes are defined in six specific planes established by the 45SW for the given mission or program. The planes are tied to the RS displays and involve two views. The top-down view is the Horizontal Plane (HP) and the side view is the Vertical Plane (VP). The HP has four envelopes for the vehicle's Instantaneous Impact Point (IIP), or location on the ground where the vehicle would fall if it were to suddenly lose power and drop in a vacuum. The HP envelopes are the right lateral/crossrange, left lateral/crossrange, maximum downrange, and minimum downrange envelopes. The VP has two envelopes for the vehicle's present position in terms of steepness relative to geographic lines drawn to protect populated areas near the launch site.

The Shuttle nominal ascent trajectory and ascent flight envelopes were delivered to the 45SW in an Ascent Data Package. The contents of this Package are listed in Table 1.

Table 1. Ascent data package contents

\begin{tabular}{|l|l|}
\hline & Delivery Letter to 45SW \\
\hline 1 & Descriptive data file for trajectory data being delivered \\
\hline 2 & Nominal Ascent Trajectory \\
\hline 3 & Launch Area Steep Vertical Plane Envelope \\
\hline 4 & Launch Area Lateral Vertical Plane Envelope \\
\hline 5 & Maximum IIP Horizontal Plane Envelope \\
\hline 6 & Minimum IIP Horizontal Plane Envelope \\
\hline 7 & Right Crossrange IIP Horizontal Plane Envelope \\
\hline 8 & Left Crossrange IIP Horizontal Plane Envelope \\
\hline 9 & Contingency (2 Space Shuttle Main Engines out at lift-off) Ascent Trajectory \\
\hline 10 & Contingency Launch Area Steep Vertical Plane Envelope \\
\hline 11 & Contingency Launch Area Lateral Vertical Plane Envelope \\
\hline
\end{tabular}

At times, the methodologies used to generate the Shuttle HP and VP envelopes have differed. However, this document does not go into this level of detail. For simplicity's sake, two of the envelopes in the Ascent Data Package for a typical nonrendezvous mission will be discussed in this document: the right and left crossrange HP envelopes. Figure 1 illustrates a typical Shuttle crossrange envelope.

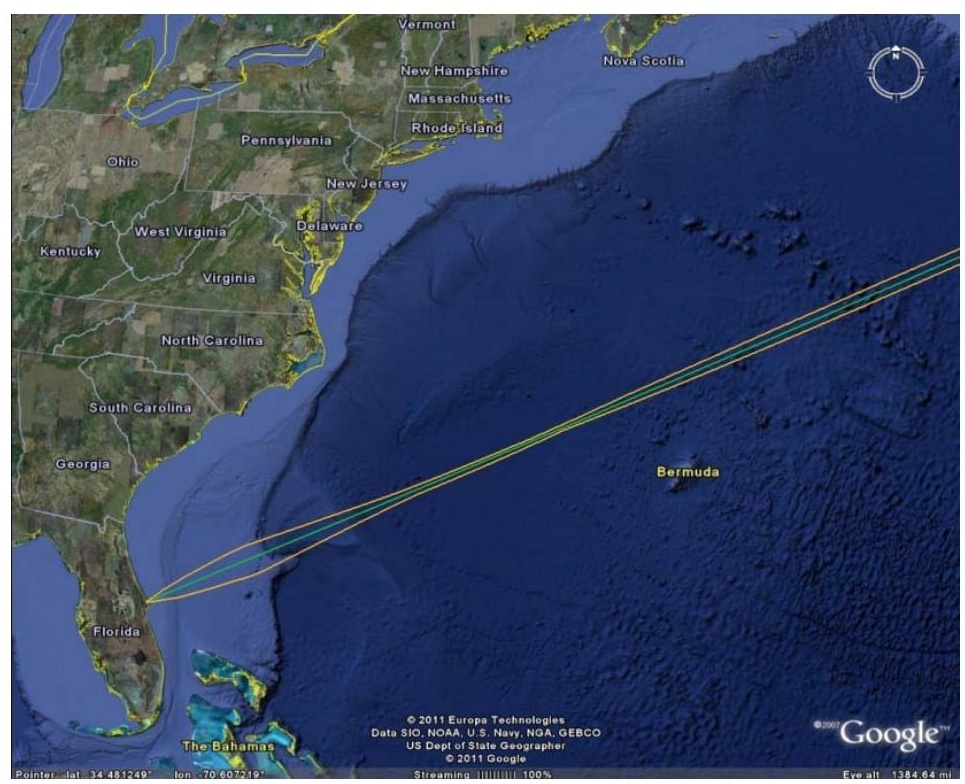

Figure 1. Example of as-flown Shuttle horizontal plane crossrange envelopes. Right and left crossrange envelopes are co-plotted with the nominal ascent trajectory for a 39 degree inclination, nonrendezvous Shuttle mission.

(Chart by Kyle Barron, United Space Alliance)

2

American Institute of Aeronautics and Astronautics Placeholder for Copyright statement. 
The requirement from 45SW for the HP crossrange envelopes is as follows:

"Three-sigma lateral trajectories define the crossrange limits of normality for the vacuum instantaneous impact point (IIP). Both a 3-sigma left and a 3-sigma right trajectory shall be provided. These trajectories should be calculated using the worst lateral wind conditions for which a launch would be attempted....”*

While the 45SW envelope requirements have remained largely unchanged for the Shuttle Program, the design of the envelopes has evolved over the years to support mission changes, maintain data quality, and reduce costs. The design of the envelopes involved both the methodology, what was technically done, and the process, how it was done. The situation of building envelopes for an operational space program with over 100 missions may or may not happen again. However, the sheer number of envelope sets designed for Shuttle allowed designers the opportunity to encounter different situations and try different approaches. This experience can provide insights helpful for future envelope generation situations. This paper describes, at a high level, the envelope concept and the evolution of the Shuttle envelope design in terms of both methodology and process.

\section{Envelope Concept}

Envelopes required by the 45SW are actual trajectory data files and not just boundary coordinates. An envelope trajectory is basically an ascent trajectory modeled appropriately to reflect the bounds of the vehicle's normal flying region considering the unknowns that could affect the trajectory's position during ascent. Shuttle unknowns include, but are not limited to, the winds of the day and the performance of various vehicle systems, such as: aerodynamics, propulsion, mass properties, Inertial Measurement Unit (IMU), Center of Gravity (CG), etc. Unknowns also include environmental uncertainties such as atmospheric data.

Unknowns represent the components, or pieces, of the total envelope. As the Shuttle Program progressed, it became necessary to incorporate more unknowns into the envelopes. There are different ways to generate envelopes, but the first step of any envelope study is to identify the ascent unknowns. There are two types of envelope unknowns: absolutes and dispersions. They are described below.

\section{A. Absolute Unknowns}

Absolute unknowns in the envelope are factors that are considered in an additive or worst-on-worst fashion because they are planned for. Examples for Shuttle included Initialization Loads (I-loads) and winds. I-loads refer to Shuttle pitch, roll, and yaw commands used by guidance to meet powered flight objectives through ascent while maintaining structural loads within constraints. These I-loads had a large influence on the ascent trajectory's position and, therefore, were a large factor in the RS envelope methodology.

The influence the winds have on an ascent trajectory is also an important component of flight envelopes. An appropriate wind that maximizes the envelope in its respective plane is required when designing an envelope. For example, the Shuttle HP crossrange envelopes used a synthetic wind file (WIND) with 3-sigma dispersed crosswind data.

\section{B. Dispersion Unknowns}

It is planned that the vehicle systems perform nominally through ascent. However, there could be variations within the "normal" range. The effect these system and environmental error sources, or dispersions, have on the ascent trajectory's position is factored into the envelopes with a 3-sigma System Dispersion (SD) component. In order to quantify the amount of trajectory movement involved with this component, an ascent dispersion analysis was conducted early in the Shuttle Program. At the time, a Monte Carlo approach that involved running a statistically significant number of dispersed trajectories with independent and random uncertainties was not feasible. Therefore, the SD effect was analyzed with a Linear Error Analysis (LEA)-type approach that involved two parts.

The first part of the dispersion analysis involved defining the 3-sigma SD component size. For the Shuttle, dispersions were identified and modeled one at a time with +3-sigma and -3-sigma tolerances. Any dispersion that was affected by monthly variations was modeled with a 3-sigma annual tolerance so that the results of the study would be annual, or applicable for a launch in any month. Data were post-processed to calculate the 3-sigma SD component size in each envelope plane.

Second, an iterative analysis was conducted to define the trajectory simulation modeling commands that force a trajectory to fly greater than or equal to the 3-sigma SD size. Once these SD elements were known, they could be used in future trajectory envelope simulations.

A beneficial aspect of running a LEA study was that individual dispersion effects were determined. This sensitivity data provided insight into how each dispersion affected the ascent trajectory's position and which dispersions had a larger influence on the trajectory. As the years progressed, the sensitivity data were also useful as

3

American Institute of Aeronautics and Astronautics

Placeholder for Copyright statement. 
a benchmark upon which to assess individual dispersion or system updates. Analyses conducted at various points in the Program, for example when the IMUs were upgraded, proved that the SD data generated early in the Program were still applicable.

This concept of having the dispersion effect as a stand-alone component of the total envelope has provided a level of flexibility to the envelope design that was helpful for the operational nature of the Shuttle Program, particularly when the envelope design became more complex with advancements in I-load design.

To summarize, there are different unknowns that could affect a vehicle's position during ascent. When defining envelopes, the bottom line is that for any ascent trajectory, regardless of I-load, wind, and launch time, a 3-sigma ascent flight envelope can be determined to represent the normal flying region of the vehicle. The various envelope methodologies used for the Shuttle Program over the years are described in the following section.

\section{Envelope Methodology}

If the Shuttle ascent trajectory design had remained unchanged throughout the program, then perhaps the same methodology would have been used to generate envelopes for the entire Shuttle Program. However, there were upgrades to the ascent trajectory design process that initiated new envelope studies. Each study required a custom methodology to build new envelopes. Consideration was also given to making the envelopes as insensitive as possible to mission updates and launch date changes, thereby minimizing the chance of an envelope redelivery and the potential for a launch slip. Each new envelope methodology plan was approved by the 45SW prior to implementation.

Table 2 provides a chronological list of the envelope studies generated for the Shuttle Program, grouped into mission-specific and generic categories. Mission-specific envelopes were unique trajectories generated for a single Shuttle mission and generic envelopes refer to one set of envelopes reused for multiple similar missions. There were five major studies and this list can be used to walk through the evolution of RS envelope design methodology.

Table 2. List of Shuttle envelope studies/methodologies

\begin{tabular}{|lcc|}
\hline \multicolumn{1}{|c|}{ Envelope Design } & First Flight & Launch Date \\
\hline Mission-specific: & & \\
Base I-load envelopes & STS-1 & 1981 \\
Alternate I-load envelopes & STS-27 & 1988 \\
DOLILU I envelopes & STS-48 & 1991 \\
\hline \multicolumn{1}{|c|}{ Generic: } & STS-70 & 1995 \\
DOLILU II envelopes & STS-88 & 1998 \\
Performance enhancement envelopes & & \\
& & \\
\hline
\end{tabular}

The first Shuttle envelope study in Table 2 (Base I-load) was the envelope study used for the early Shuttle missions. The title Base I-load refers to the I-load design process used for missions until 1988. The next three envelope studies (Alternate I-load, Day-of-Launch I-load Update (DOLILU) I, and DOLILU II) were initiated because of a change to the Shuttle I-load design process. Similarly, these envelope study titles refer to the appropriate I-load design process because that is what drove the methodology change.

I-load design methodology changed from a preflight design based on a preflight estimated wind to a Day-Of-Launch (DOL) design which took the actual real-time winds into account. Preflight I-load designs had a specific time period, or launch season, for which they applied, ranging from one month to a series of months. When envelopes were redesigned, the methodology took the launch season into account. Other envelope inputs were adjusted to account for the launch season so the resulting envelopes could be valid for as long as possible. Having this flexibility in the envelope design reduced the likelihood of a redelivery if the launch date moved.

The current and final RS envelope study is the Performance Enhancement (PE) envelope study listed in Table 2. This study was initiated when Shuttle missions were manifested to build the International Space Station (ISS). Ascent design changes were necessary to allow the Shuttle to transport heavier payloads to the ISS. The changes involved hardware and software modifications, referred to as PEs, and included options for two different Main Engine Cutoff (MECO) target altitudes, two different types of Space Shuttle Main Engines (SSMEs), and multiple

4

American Institute of Aeronautics and Astronautics

Placeholder for Copyright statement. 
throttle settings, among others. For each ISS mission, the appropriate combination of PEs was used to meet unique mission objectives; therefore the envelope design had to account for any PE combination. The RS envelope design for these types of missions involved envelope trajectories that applied for a launch in any month of the year ("annual") and could be reused for similar missions ("generic"). Since 1998, the same generic envelope trajectories have been used for all ISS missions.

The following section chronologically walks through all of these five envelope methodologies in regards to generating Shuttle HP crossrange envelopes for a nonrendezvous mission.

\section{A. Mission-Specific Envelopes}

Mission-specific envelopes, used for the first half of the Shuttle Program, were defined by envelope components or sizes that were applied to a given mission's nominal ascent trajectory in each plane. Because each mission's preflight nominal trajectory was unique, the corresponding envelopes flew over a different area in the ocean flightto-flight.

\section{Base I-load Envelopes}

At the beginning of the Shuttle Program, first-stage I-loads, referred to as base I-loads, were designed months before the scheduled launch. The base I-load set was applicable for the expected launch month. When launch day arrived, if the DOL winds yielded an acceptable DOL trajectory with respect to loads and ascent constraints, then launch was possible that day. If not, then there was no option to redesign the nominal I-loads and the launch was postponed to another day.

The envelope components for these base I-load missions were rather straightforward. The ascent unknowns were how the vehicle systems would perform, the SD effect, and what the wind would be on launch day, the WIND effect. The mission-specific envelopes for each plane were created by running the preflight nominal ascent trajectory with the appropriate SD element and WIND file. (See Figure 2.) Launch slips to another month meant the envelopes were regenerated.

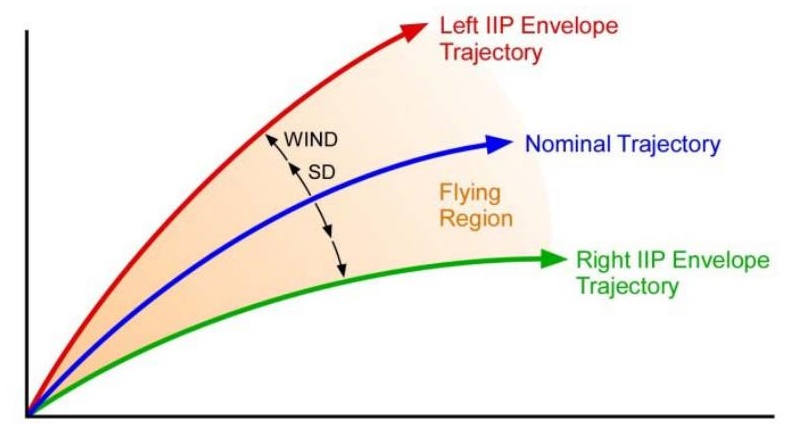

Figure 2. Envelope concept for typical HP crossrange base I-load mission envelope

\section{Alternate I-load Envelopes}

In order to increase the chance of having I-loads acceptable for the DOL wind, the alternate I-load design was established in 1988. This design involved expanding the base I-load design to also allow for four, and later eight, alternate sets of I-loads to be made available on launch day. After the winds were measured on launch day, the base or alternate I-load set that brought the DOL trajectory back to the preflight nominal was selected for uplink to the Orbiter. The I-loads were designed for a given launch season which involved a series of months.

Conceptually, the envelope design for alternate I-load missions remained similar to the base I-load design. Envelopes were generated by running a preflight nominal trajectory with a SD element and a WIND file. However, instead of having one preflight nominal trajectory, there were either five or nine trajectories that were tied to the sets of I-loads available on launch day. To determine which preflight nominal trajectory to use to build the envelopes, all nominal trajectories were plotted in each envelope plane. The most extreme trajectory for each plane was selected. For the left crossrange envelope, the trajectory that flew the most left in the HP was selected. (See Figure 3.) Because the I-loads for this design accounted for a launch season, the WIND data were generated to account for this season. 


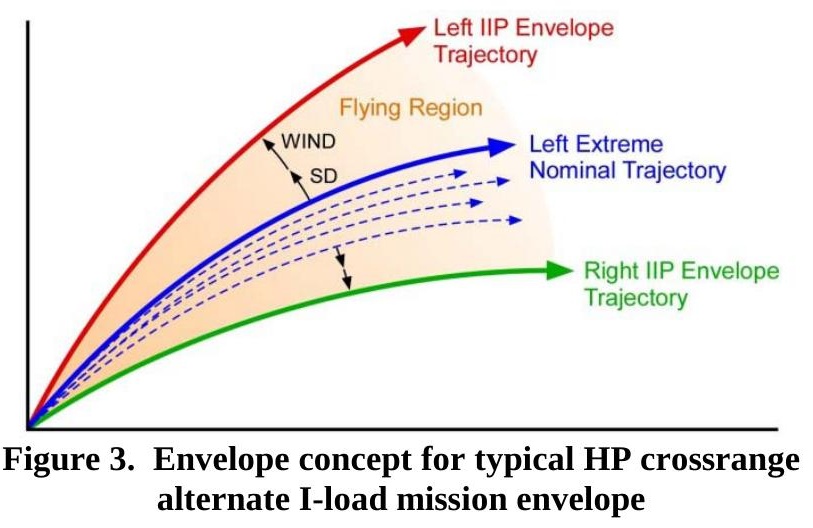

The resulting mission-specific envelopes accounted for any of the alternate I-load sets being chosen on launch day. They were applicable for the launch season set by the I-load design. A launch slip outside the season required envelopes to be regenerated.

\section{DOLILU I Envelopes}

In 1991, the I-load process referred to as DOLILU I was implemented. This process represented a significant change to the way Shuttle first-stage I-loads were designed. A preflight designed I-load set was still available for use on launch day. However, for the first time a new I-load design could be conducted hours before launch utilizing the actual DOL winds. DOLILU I I-loads were designed with a software tool to anticipate the DOL wind and bring the trajectory back to the preflight-nominal trajectory. In order to determine which I-load set to fly with on launch day, vehicle loads were assessed for both the DOLILU I I-load set and the preflight I-load set. The better I-load set with respect to launch probability was chosen for flight.

The DOLILU I design produced first stage in-plane and out-of-plane steering effects that altered the vehicle's ascent trajectory position and, therefore, needed to be addressed in the RS envelope study. A composite envelope was designed that took into account both a non-DOLILU I-load envelope and DOLILU I-load envelope such that any I-load used on DOL would be accommodated.

a) First component: non-DOLILU envelope

The non-DOLILU envelope was built in a similar fashion to those in the past. The extreme nominal trajectory for the I-load season was run with a SD element and WIND file.

b) Second component: DOLILU envelope

Considering the DOL I-load design approach, it was no longer straightforward to select an extreme/left nominal trajectory for envelope generation because there were many I-load possibilities and they would not be known until launch day. Therefore, the envelope concept changed to an "envelope target" design where component sizes could be combined and applied to a nominal reference trajectory to define the envelope flying region.

For the DOLILU envelope, the following unknowns were identified: the SD effect, the Wind Persistence (WP) effect, and the DOLILU effect that accounts for the DOLILU I I-loads and winds. The SD component size was carried over from the previous analysis. The two new components, WP and DOLILU, were assessed in the DOLILU I envelope study using representative wind data. They are described below. The WP effect became necessary because it was not possible to perform the DOLILU design process instantaneously at lift-off. The DOL I-loads were built hours prior to launch and it was expected that the DOL measured wind would change between the time the I-loads were designed to when they were flown. Therefore, the trajectory position would be slightly different than the one predicted.

The DOLILU effect accounted for the range of trajectory movement possible in each envelope plane for a DOLILU trajectory considering DOL I-loads and winds. It is important to note the difference between how winds were accommodated in a DOLILU and non-DOLILU envelope. For a DOLILU envelope, the DOLILU effect accommodated the trajectory movement possible by having I-loads built on DOL using the winds of the day. Therefore, numerous DOL wind files were used to accommodate winds expected on launch day. For a non-DOLILU envelope, the WIND effect accounted for the trajectory movement possible by having a base preflight I-load set flown with a dispersed statistical wind that maximized the envelope in a given plane. 
Once the three envelope components were available, they were added to the reference nominal trajectory to obtain the DOLILU flight envelope.

c) Composite result: Total envelope

After a non-DOLILU envelope and a DOLILU envelope were generated in each plane, they were co-plotted for comparison. The DOLILU envelope was extreme for the first $\sim 65$ seconds of ascent and the

non-DOLILU envelope was extreme after that. The reason was that the DOLILU trajectory was designed to fly into the DOL measured wind in such a manner as to eliminate load exceedances in the high loads region and ensure that certain conditions at Solid Rocket Booster (SRB) separation are met. For example, a left crosswind would cause the early DOLILU trajectory to steer to the left.

Therefore, for the final envelope, it was decided to use the composite of the non-DOLILU and DOLILU envelopes. The results of the DOLILU I envelope study were a seasonal envelope size for each plane that could be applied to the extreme mission-specific nominal trajectory.

For DOLILU I missions, the extreme mission-specific nominal trajectory was run with the SD element, the WIND file, and, for the first time, artificial trajectory adjustments. These adjustments involved altering simulation input data and/or I-load parameters to yield a trajectory that tracked greater than or equal to the composite envelope size. (See Figure 4.)

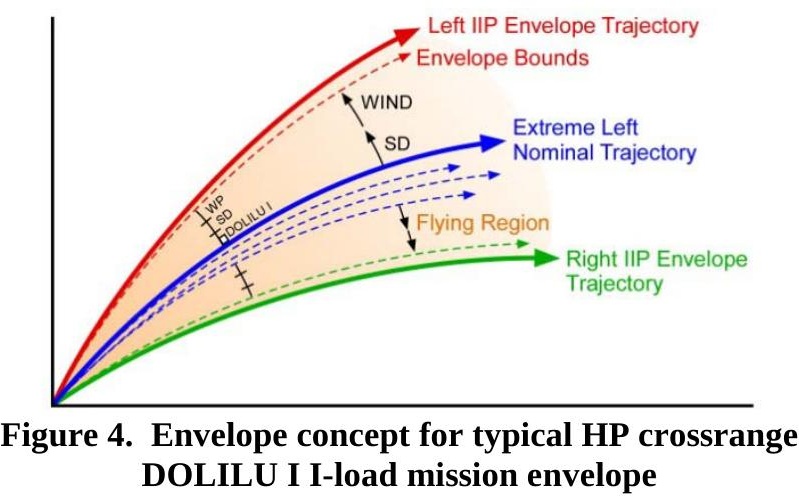

The final mission-specific envelopes delivered to the 45SW for DOLILU I missions were limited in applicability to the I-load design season. A launch slip outside this season would require new envelopes.

\section{B. Generic Envelopes}

Over time, it became cost-effective for the Shuttle Program to use generic, or reusable, envelopes. Saving costs in the flight-to-flight envelope production area freed up resources in the RS group to be available to support other RS activities.

RS envelope studies that generated generic envelopes were also able to accommodate annual inputs such that the resulting generic envelopes were available for a launch in any month. This was also important for the operational Shuttle Program as launch date and manifest changes were common. In this document, the term "generic envelopes” refers to envelopes that are both generic and annual.

Generic envelopes represent actual vehicle trajectory limits independent of where the nominal reference trajectory flies because the nominal trajectory variation is built into the envelope. This type of envelope can be viewed as a fixed geographical area over the ocean where the envelope flies greater than or equal to the bounds defined in an envelope study.

\section{DOLILU II Envelopes}

From an ascent trajectory design standpoint, the DOLILU II upgrade involved the DOL I-load design changing from a guidance parameter update to a guidance and flight control parameter update. On DOL, the wind was measured and first-stage pitch/yaw I-loads, as well as throttle settings, were designed and uplinked to the Orbiter's onboard computers for use during ascent. Unlike DOLILU I, where the I-loads were designed to bring the DOL trajectory back to the preflight nominal, the DOLILU II process did not reference a preflight nominal trajectory. Instead, the DOLILU II process built I-loads that resulted in a trajectory that balanced dynamic loads and eliminated load exceedances. 
Of importance to RS was the fact that a mission-specific preflight nominal ascent trajectory was no longer available with I-loads suitable for flight. This had two impacts to the RS envelope design:

1) The only I-loads that the envelope design had to account for were the DOLILU II I-loads. No longer was a preflight I-load set, tied to a launch season, part of the envelope design. Therefore, it became possible to make envelopes annual for the first time.

2) The total envelope needed to account for where the DOLILU II nominal trajectory could be on launch day. Therefore, it was necessary to quantify the swath of space over the ocean subject to a possible DOL trajectory. From this swath the other envelope components could be applied such that a total envelope area, a fixed area over the ocean, could be defined. In order to define envelopes in this fashion, an anchor trajectory was necessary from which to make all envelope measurements. This methodology approach was used for the DOLILU II and PE envelope studies.

To start the DOLILU II envelope study, the envelope unknowns were identified: DOLILU II effect (I-loads and winds), SD effect, WP effect, and Mission Weight (WT) effect. The SD and WP effects were borrowed from the previous envelope study as they were unaffected by the new DOLILU II I-load design. Information concerning the new DOLILU II and WT effect components is provided below.

Unlike the DOLILU I study, where the DOLILU I effect was generated to represent the trajectory variation with respect to a monthly mean wind reference case, the DOLILU II effect was generated to define the total area possible for a DOLILU II nominal trajectory. Representative wind data were used in the DOLILU II envelope study to define the DOLILU II component. A trajectory was pulled from the DOLILU II component assessment to use as the "anchor" trajectory for the study. All envelope component sizes were measured against this anchor trajectory.

The WT effect was assessed in the DOLILU II envelope study and took into account the range of total mission weights possible.

The total DOLILU II envelope size in each plane was determined by adding each component size (DOLILU II, SD, WP and WT) to the anchor trajectory to produce the total envelope bounds. These bounds represented the fixed geographic envelope, as illustrated in Figure 5.

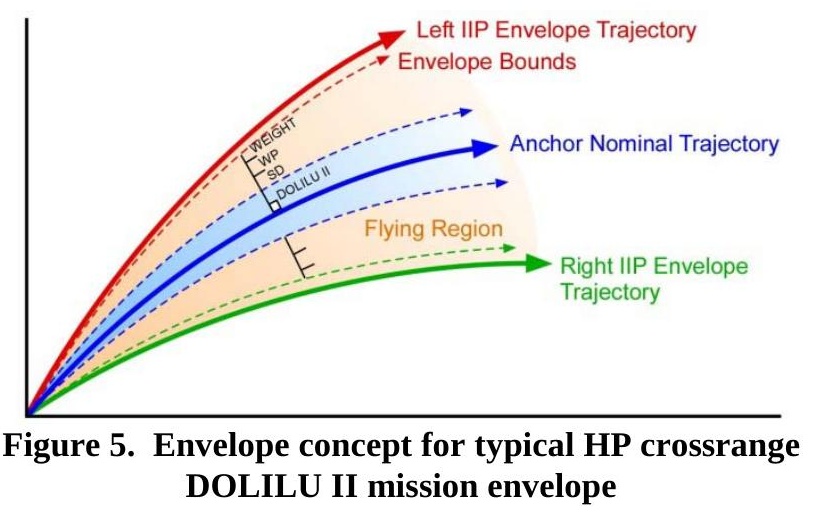

For the first Shuttle DOLILU II mission, generic right and left envelope trajectories were generated that flew greater than or equal to the bounds. For subsequent flights, mission data were assessed against the DOLILU II envelope study groundrules to ensure the generic trajectories applied. Following this short verification, the remainder of the Ascent Data Package was built, only this time the number of trajectories in the Package was reduced. Notification was placed in the Package delivery letter instructing the $45 \mathrm{SW}$ to use previously-delivered generic envelopes for the mission.

\section{Performance Enhancement Envelopes}

The PE envelope study produced generic envelope bounds for the later portion of the Shuttle Program. For this design, the DOLILU II process was still used to generate I-loads. However, the Shuttle ascent design change that forced the envelope methodology update this time was not due to I-loads, but rather to PEs. PEs affected the ascent trajectory profile and, therefore, a new envelope study was required.

For each ISS mission after 1997, the appropriate combination of PEs was used to meet unique mission objectives. Therefore, to allow generic envelopes to be applicable for multiple ISS missions, additional mission parameters beyond mission weight had to be considered. Now, a PE effect had to be included to accommodate any combination of PEs selected for a given ISS mission. 
The complete set of envelope unknowns for PE envelopes were: PE/DOLILU II effect, SD effect, WP effect, and PE mission parameter effect. The SD effect and WP effect were carried over from the previous envelope study as they were not affected by the change to PEs. The other components were assessed in the PE envelope study. To conclude the analysis, all envelope components were combined and applied to the anchor trajectory to define the fixed geographic envelope bounds for PE missions. (See Figure 6.)

As was done for the previous envelope study, the RS product generation process for the first PE flight involved finding trajectories that flew greater than or equal to the right and left crossrange generic bounds. Once these trajectories were found, they became the generic envelope trajectories applicable for future similar missions with mission-specific parameters covered by the PE envelope study groundrules.

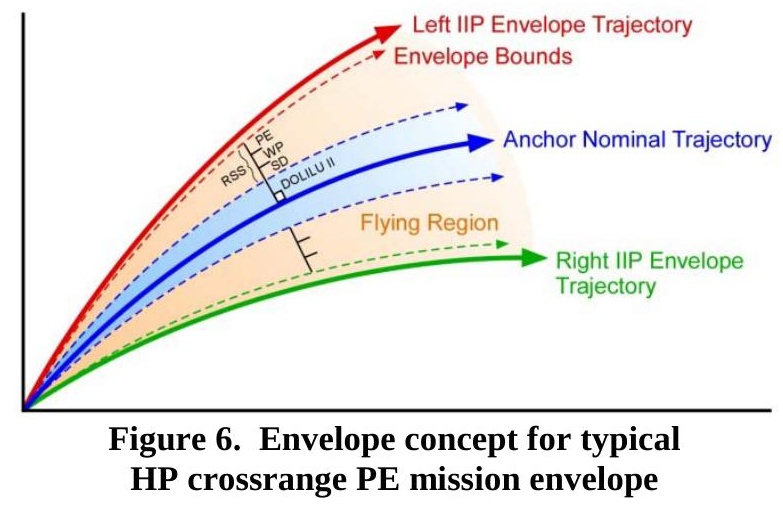

Towards the later portion of the Shuttle Program when only ISS missions were flown, the Ascent Data Package looked quite different than it did early in the program. Not only were the HP crossrange envelopes generic, but all other envelopes were as well. Therefore, the Package was much smaller, consisting of only two trajectory data files. (See Table 3.)

Table 3. Ascent data package contents-later Shuttle Program

\begin{tabular}{|l|l|}
\hline & Delivery Letter to 45SW \\
\hline 1 & Descriptive data file for trajectory data being delivered \\
\hline 2 & Nominal Ascent Trajectory \\
\hline 3 & Contingency Ascent Trajectory \\
\hline
\end{tabular}

\section{Envelope Design Process Support}

Early in the Program, envelopes were made specifically for each mission by the RS group with a relatively simple process. Engineers would generate the data using informal procedures and a relatively simple suite of software processing tools. Many of the required data generation inputs were entered manually at the command line. New employees were trained via on-the-job training and learned from experienced engineers.

Throughout the years, many process improvement initiatives were implemented from the company, department, and RS group levels. The goals were to

- Maintain data quality

- Standardize the product generation process so it was consistent and repeatable for each flight

- Reduce the cycle timeline and data generation costs

- Increase customer satisfaction

There were two envelope design processes, each tied to the type of work required to support the RS envelope requirement: a production process and an analysis process. The production process was used to generate products or consistent data required for each mission with a defined process and timeline. Analyses or studies were required for new requirements or to accommodate mission/hardware changes into existing requirements. Analyses involved a nondefined process, as the methodology was built for the given situation and the timeline was dictated by events. For example, when PEs were implemented there was a PE envelope analysis that determined appropriate methodology. For each PE mission afterwards, envelope products, with a reference to generic envelopes, were generated as part of the normal, defined RS production process.

9

American Institute of Aeronautics and Astronautics

Placeholder for Copyright statement. 
For simplicity, this document describes the process evolution for envelope products only. This section describes how the envelope generation process has improved in the areas of: Configuration Management (CM), employee training, Quality Assurance (QA), and process automation. Unlike the methodology discussion, this information is not chronological.

\section{A. Configuration Management}

A CM effort was implemented at the department level in order to protect the integrity of data, software, and procedures used to generate Shuttle ascent trajectory products. This effort resulted in a document that outlined the department's policies related to CM, which came to be known as "the CM plan." In regards to envelope design, the RS group initially captured the data, software, and procedures used to build the envelopes and baselined them in accordance with the CM plan. The plan involved a lifecycle process to verify that the data requirements, implementation of requirements, and testing were correct. Then the information was locked down in a consistent location for use. Once this solid foundation was obtained, future additions or changes to the information were forwarded through the plan.

By having this CM plan, the process used to generate RS envelope products was standardized, thereby being consistent and repeatable for each mission.

\section{B. Employee Training}

As with the CM plan, the employee training effort was initiated on a department-wide scale. The long history of building trajectory data for the Shuttle Program made it important that information be captured for future employees. The RS group developed its training program and focused on the following training goals:

- Train employees as efficiently as possible so they could begin work quickly

- Allow the group to emphasize training areas, as needed, to meet group goals and workload expectations

- Reduce the time senior engineers spent training new hires

- Capture knowledge before employee turnover occurred

The training program for RS product generation involved providing the employee with the knowledge necessary to generate a RS product. A training flow and certification criteria were established which outlined the steps a new hire would take to become certified in product generation. The tasks included reading training manuals written by the group, generating practice flight data, and working with senior engineers. New employees were assigned a mentor who was a more experienced member of the group. As a capstone activity, each trainee answered a list of questions to verify that he/she had satisfactorily learned the knowledge required for each training task. Upon sign-off of all training criteria, the employee was deemed certified and able to work on a flight envelope product.

\section{Quality Assurance}

Over the course of the Shuttle Program, there has been a thorough QA process to ensure all data were correct prior to delivery to customers. Over the years, the process has matured and strengthened. For RS products, the product QA process involved three different trained employees verifying the data

a) Product generator - generator QA

b) Fellow product generator - peer QA

c) Experienced engineer approved by management - lead QA

Each level of QA ensured the product was generated with the correct process, accurately reflected the given mission, and adhered to 45SW data requirements. The improvements to the QA measures over the years involved lead QA training, CM QA checklists, and practice training flights with hardwired errors. The checklists were included with the RS procedures and were managed by the CM plan.

\section{Process Automation}

Over the years, ways to automate the envelope generation process were implemented in order to reduce costs and maintain quality. Where it made sense, data contents were streamlined and software was automated.

Product streamlining involved having good customer requirements and meeting the requirements efficiently. Periodically, the requirements for the data products were discussed with the customers. It was helpful to confirm that the requirements were still current and that the product still met customer expectations. In addition, the development of generic envelopes was a large factor in streamlining envelope data. By not having to generate mission-specific envelopes for each mission, cost savings were obtained and the chance for a data error was reduced. Generic data also greatly reduced the chance of a data redelivery in the event of certain mission changes.

10

American Institute of Aeronautics and Astronautics

Placeholder for Copyright statement. 
Software automation was implemented to reduce the likelihood of data entry errors and to reduce the time and resources required to build the products. Later, when generic envelopes were used, the software was still helpful when generating the nominal ascent trajectory portion of the Ascent Data Package. The software maintenance impact was taken into account when working on software automation. Overall, it was very effective to have software automation at certain points in the process.

\section{Summary of RS Envelope Design Evolution}

The envelope design for the early Shuttle missions involved a rather straightforward methodology and process. The envelopes were sensitive to certain mission changes. Over time, the envelope methodology changed to incorporate flight design updates. For each new study, consideration was given to making the envelopes as insensitive as possible to changes so that the risk of a data redelivery was minimized.

At the end of Program, the envelopes were generic. The chance for errors was reduced because data were not generated for each mission. The generic envelopes were flexible because they could accommodate launch slips, many mission weight updates, and launch pad changes. Resources were saved by not having to generate, QA, and deliver envelopes for each mission. Instead, all that was required was a short verification process to ensure mission data fell within envelope study groundrules. By having generic envelopes that minimized the chance of a data redelivery, costs were also saved at the 45SW because the chance of having to update display data was also minimized.

From a big picture standpoint, the RS Ascent Data Package was only one part of the total RS group responsibilities for the Shuttle Program. The operational nature of the Shuttle Program meant that the RS group produced repeatable data deliverables as well as unique analyses to accommodate Program changes/improvements. By having a mature envelope methodology and process, resources were saved in the production area and made available for other tasks.

To summarize the design evolution, Figure 7 provides a snapshot of the Shuttle envelope design at the beginning and end of the Program. Although there were more envelope unknowns later in the Program, the deliverable Ascent Data Package was a much smaller product due to the stability of the Shuttle mission types and the design of the envelopes. 


\section{Space Shuttle Ascent Flight Envelope Design}

\section{Early Shuttle Program}

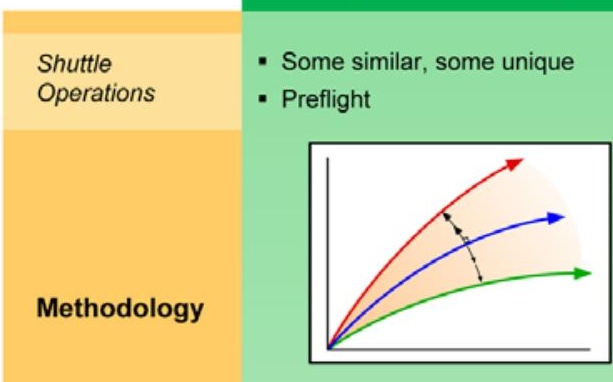

- Variable location

- Mission specific

- Redelivery required for launch slips out of design season

- Sensitive to mission changes

\section{Late Shuttle Program}

Mission Types

I-Load Design

- Mostly constant (ISS)

- Day of launch

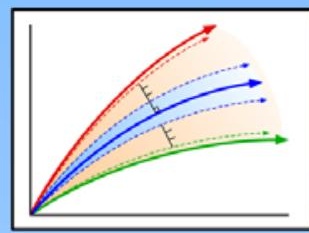

- Fixed

- Generic

Envelope - Applicable for launch in any month

Characteristics

- Less sensitive to mission changes

\begin{tabular}{|c|c|c|c|}
\hline \multirow{7}{*}{ Process } & Informal/Manual & & Standardized/Automated \\
\hline & - Employee-made and distributed & Procedures & $\begin{array}{l}\text { - Locked/managed with CM plan } \\
\text { - Standardized/repeatable process }\end{array}$ \\
\hline & - Informal & Data & - Locked/managed with $\mathrm{CM}$ plan \\
\hline & - Modified by engineers & $\begin{array}{c}\text { Software } \\
\text { Processing Tools }\end{array}$ & - Locked/managed with CM plan \\
\hline & - On-the-job training & Employee Training & $\begin{array}{l}\text { - Certification plan for each } \\
\text { employee }\end{array}$ \\
\hline & $\begin{array}{l}\text { - } 3 \text { levels of QA } \\
\text { - } \quad \text { Informal checklists }\end{array}$ & Quality Assurance & $\begin{array}{l}\text { - } 3 \text { levels of QA } \\
\text { - } \quad \text { QA training and CM checklists }\end{array}$ \\
\hline & $\begin{array}{l}\text { - Existing requirements } \\
\text { - Suite of software tools }\end{array}$ & Automation & $\begin{array}{l}\text { - Scrubbed requirements } \\
\text { - Software automation tool }\end{array}$ \\
\hline
\end{tabular}

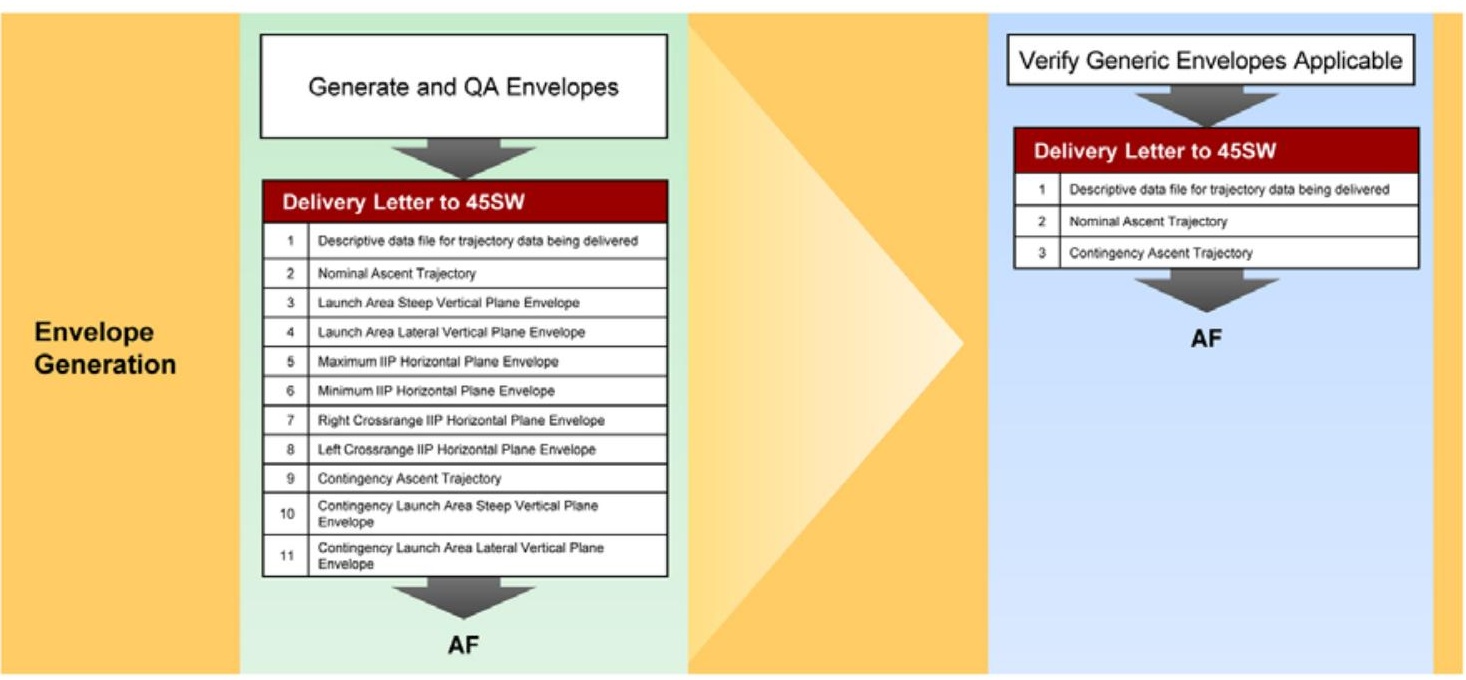

Figure 7. Evolution of Shuttle ascent flight envelope design 


\section{Conclusion}

The Shuttle envelope design started as a manual process building mission-specific envelopes and evolved into an automated process reusing generic envelopes. During this time, necessary envelope modifications were made to accommodate Shuttle ascent design changes. The history of on-time, error-free envelope product generation has contributed to mission success for the Shuttle Program and provided lessons learned that can contribute to future spaceflight endeavors.

\section{Acknowledgments}

The authors would like to thank Christina Harmom, Charles Brown, and Erica Berry-Lozano for their support and contributions. Also, thanks to Kyle Barron for the illustration in Figure 1, Jennifer Hees for all other graphics, and Debra Dallas, Rick Dilworth, Jonathan Daugbjerg, and Patricia Vergil for assistance with the formatting and graphics.

$\begin{array}{ll} & \\ \text { 45SW } & =45^{\text {th }} \text { Space Wing } \\ \text { CG } & =\text { Center of Gravity } \\ \text { CM } & =\text { Configuration Management } \\ \text { DOL } & =\text { Day-of-Launch } \\ \text { DOLILU } & =\text { Day-of-Launch I-Load Update } \\ \text { ER } & =\text { Eastern Range } \\ \text { HP } & =\text { Horizontal Plane } \\ \text { IIP } & =\text { Instantaneous Impact Point } \\ \text { I-load } & =\text { Initialization load } \\ \text { IMU } & =\text { Inertial Measurement Unit } \\ \text { ISS } & =\text { International Space Station } \\ \text { JSC } & =\text { Johnson Space Center } \\ \text { LEA } & =\text { Linear Error Analysis } \\ \text { MECO } & =\text { Main Engine Cutoff } \\ \text { NASA } & =\text { National Aeronautics and Space Administration } \\ \text { PE } & =\text { Performance Enhancement } \\ \text { QA } & =\text { Quality Assurance } \\ \text { RS } & =\text { Range Safety } \\ \text { RSS } & =\text { Root Sum Squared } \\ \text { SD } & =\text { System Dispersion } \\ \text { SRB } & =\text { Solid Rocket Booster } \\ \text { SSME } & =\text { Space Shuttle Main Engine } \\ \text { SSP } & =\text { Space Shuttle Program } \\ \text { VP } & =\text { Vertical Plane } \\ \text { WP } & =\text { Wind Persistence } \\ \text { WT } & =\text { Mission Weight } \\ & \end{array}$

\section{Reference}

*Air Force Space Command, Air Force Space Command Manual 91-710, Range Safety User Requirements Manual, Volume 2- Flight Safety Requirements, http://www.e-publishing.af.mil/, July 2004. 


\section{Evolution of Space Shuttle Range Safety Ascent Flight Envelope Design}

Purpose of Range Safety

The purpose of Range Salety is to minimizize the risk to public life, health, and property associated

with the potential hazards of launching a vehicle into space

Range Safety measures include

- Monitor vehicle launches on displays

Range Safety Support for Shuttle Missions

The NaSA Space Shuttle Program leunched is

Complex 39 at the Eastern Range in Florida

The Air Force 45th Space Wing (455W) operates these ER launch pads. Range users are required to

deliver mission data as specified in “Air Force Space Command Manual 91-710, volume 2

(AFSPCMAN 91-710)"

-Flight Envelopes are one requirement

RS Ascent Flight Envelopes

Envelopes are 3-sigma dispersed traj

from lift-off to the end of powered fight

- The displays are used to monitor the Shuttle's ascent trajectory to see if it flies as expected

displars

Delivered to 45SW in an RS Ascent Trajectory Data Package for each flight

- Account for unknowns during ascent

-System/environmental dispersions and winds

-Additional unknowns added later in the Shuttle Program to make the envelopes generic

- Five major envelope studies have been conducted for the Shuttle Program

-

- Consideration given to making the envelopes as insensitive as possible to changes

Horizontal Plane Crossrange Envelope

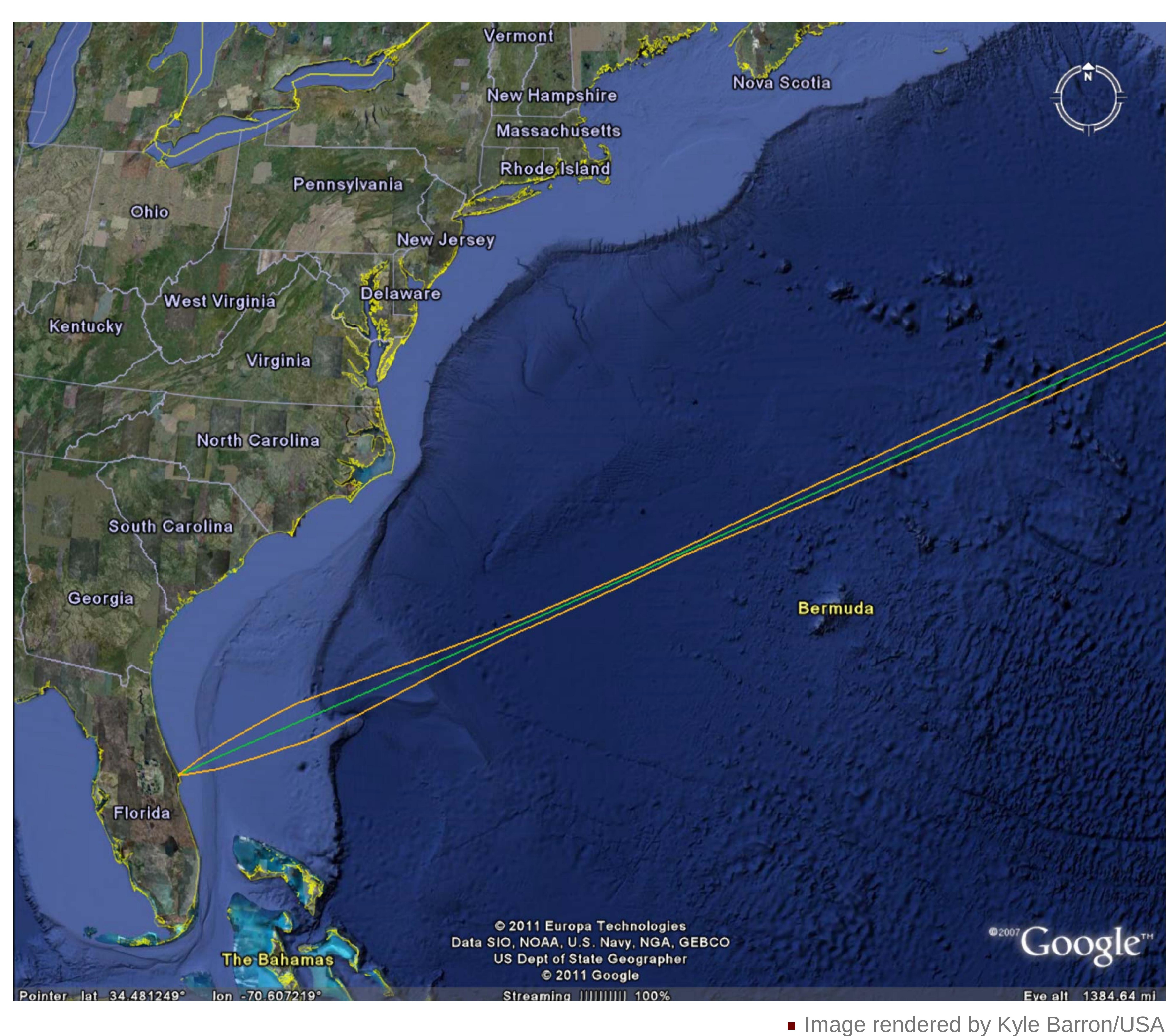

nvelope Design Evolution - Space Shuttle Ascent Flight Envelope Design

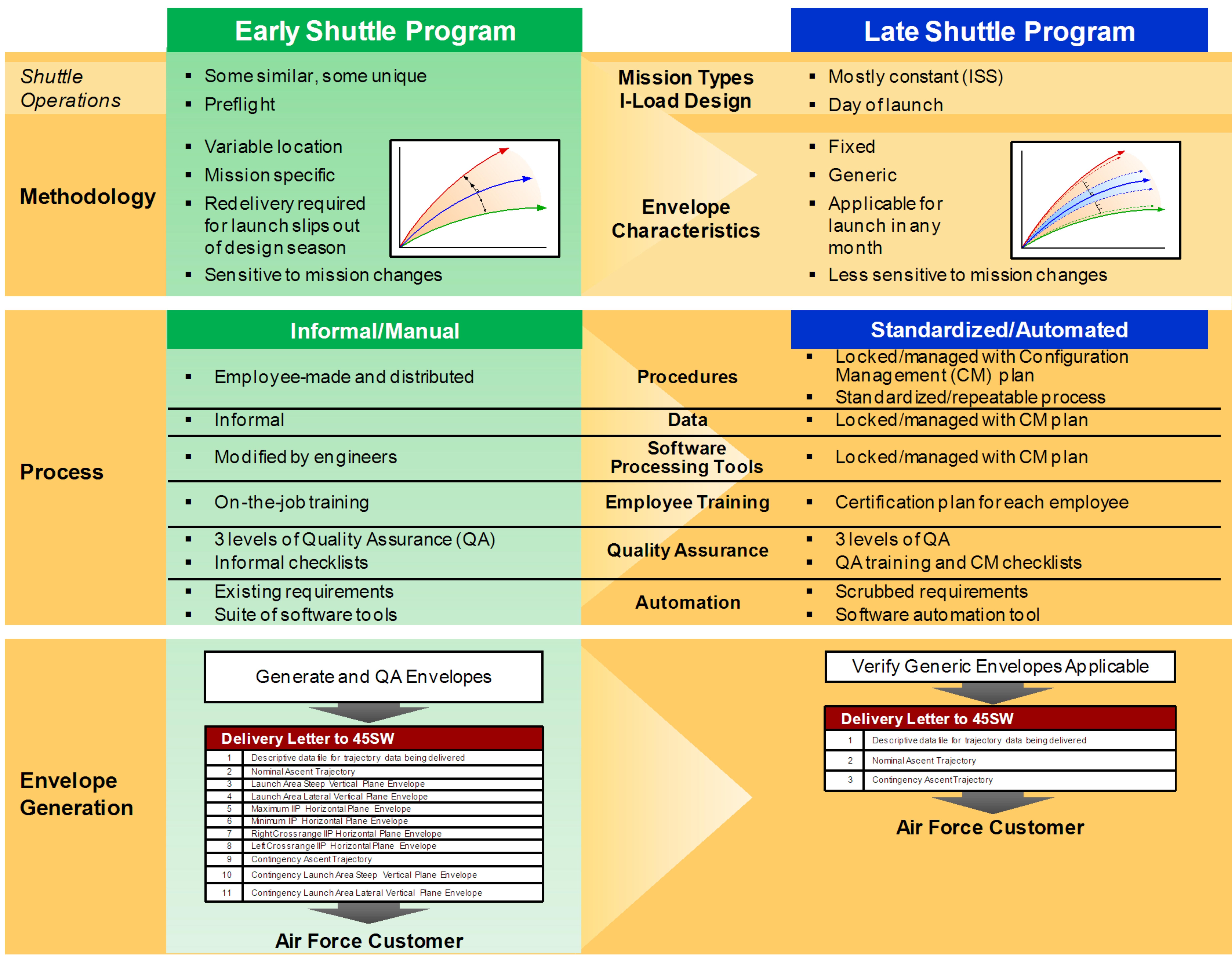

